\title{
A 4-Mid-Node Plane Model of Base Force Element Method on Complementary Energy Principle
}

\author{
Yinghua Liu, ${ }^{1}$ Yijiang Peng, ${ }^{2}$ Lijuan Zhang, ${ }^{2}$ and Qing Guo ${ }^{2}$ \\ ${ }^{1}$ AML, Department of Engineering Mechanics, Tsinghua University, Beijing 100084, China \\ ${ }^{2}$ Key Lab of Urban Security \& Disaster Engineering, Department of Civil Engineering, Beijing University of Technology, \\ Beijing 100124, China
}

Correspondence should be addressed to Yijiang Peng; pengyijiang@bjut.edu.cn

Received 18 July 2013; Accepted 12 August 2013

Academic Editor: Song Cen

Copyright (C) 2013 Yinghua Liu et al. This is an open access article distributed under the Creative Commons Attribution License, which permits unrestricted use, distribution, and reproduction in any medium, provided the original work is properly cited.

\begin{abstract}
Using the base forces as fundamental variables to describe the stress state and the displacement gradients that are the conjugate variables of the base forces to describe the deformation state for the two-dimensional elasticity problems, a 4-mid-node plane model of base force element method (BFEM) based on complementary energy principle is proposed. In this paper, the complementary energy of an element of the BFEM is constructed by using the base forces. The equilibrium conditions are released by the Lagrange multiplier method, and a modified complementary energy principle described by the base forces is obtained. The formulation of the 4-mid-node plane element of the BFEM is derived by assuming that the stress is uniformly distributed on each edge of the plane elements. A procedure of the BFEM on complementary energy principle is developed using MATLAB language. The numerical results of examples show that this model of the BFEM has high precision and is free from mesh sensitivity. This model shows good performances.
\end{abstract}

\section{Introduction}

The finite element method based on the assumed displacement field has become a method of choice for the solution of a wide variety of problems in structural mechanics. The advantages of the finite element method include the efficient and accurate modeling of domains with complex geometric configurations and varying material properties and the capability of accurately analyzing both geometrically and materially nonlinear problems. Shortcomings of the displacement model of FEM have been observed in the analyses of certain classes of problems, such as the large deformation, the treatment of nearly incompressible materials, the bending of thin plates, and the moving boundary problems. The developments of finite elements and corresponding formulations have been a subject of extensive research, which have been summarized in textbooks by Zienkiewicz [1], Bathe [2,3], and Cook [4].

The attempts to extend and generalize the complementary energy methods that have been proposed over the last almost 50 years for small deformation solid mechanics problems have led to the development of several complementary energy principles and corresponding finite element models for nonlinear elastic solid/structural mechanics problems [5-7].

Recently, based on the complementary energy principle, a hybrid stress-function element method is proposed by $\mathrm{Fu}$ et al. [8] and Cen et al. [9-12] using the mesh distortion immune technique for developing plane 8-node elements. It starts from the principle of minimum complementary energy and employs the fundamental analytical solutions of the Airy stress function as the trial functions (analytical trial function method). So as long as the element edges keep straight, the resulting 8-node element HSF-Q8 can also produce the exact solutions for quadric displacement fields, and even the element shape degenerates into triangle and concave quadrangle.

A new concept was introduced by Gao [13], who used the concept of the "base forces" to replace various stress tensors for the description of the stress state at a point. These base forces can be directly obtained from the strain energy. For large deformation problems, when the base forces were adopted, the derivation of basic formulae was simplified by Gao $[14,15]$. Based on the concept of the base forces, precise expressions for stiffness and compliance matrices for the FEM 
were obtained by Gao [13]. The applications of the stiffness matrix to the plane problems of elasticity using the four-edge plane element and the polygonal element were researched by Peng et al. [16]. Using the concept of base forces as state variables, a three-dimensional formulation of base force element method (BFEM) on complementary energy principle was proposed by Peng and Liu [17] for geometrically nonlinear problems. And the new finite element method based on the concept of base forces was called the base force element method (BFEM) by Peng and Liu [17]. A three-dimensional model of base force element method (BFEM) on complementary energy principle was proposed by Liu and Peng [18] for elasticity problems. The application of $2 \mathrm{D}$ base force element method (BFEM) to geometrically nonlinear analysis was proposed by Peng et al. [19].

The objective of the present research is to present a new two-dimensional formulation of the base forces element method (BFEM) for elasticity problems. A 4-mid-node plane element model of the BFEM for elasticity problems will be derived. In the present formulations of the BFEM, the "base forces" are treated as unknown variables, and the basic equations are constructed by means of the complementary energy principle. The element equilibrium conditions are fulfilled using the Lagrange multiplier method. Explicit expressions for the control equations are provided, and a procedure of the present method is developed. A number of elasticity problems are solved using the present formulation, and the results are compared with corresponding analytical solutions.

\section{Basic Equations}

Consider a two-dimensional domain of solid medium, let $\mathbf{x}^{\alpha}$ $(\alpha=1,2)$ denote the Lagrangian coordinate system, where $\mathbf{P}$ and $\mathbf{Q}$ are the position vectors of a material point before and after deformation, respectively. Two triads for original and current configurations can be defined as

$$
\mathbf{P}_{\alpha}=\frac{\partial \mathbf{P}}{\partial x^{\alpha}}, \quad \mathbf{Q}_{\alpha}=\frac{\partial \mathbf{Q}}{\partial x^{\alpha}} .
$$

Let $\mathbf{u}$ denote the displacement of a point; then

$$
\mathbf{u}=\mathbf{Q}-\mathbf{P} .
$$

The gradient of displacement $\mathbf{u}_{\alpha}$ can be written as

$$
\mathbf{u}_{\alpha}=\frac{\partial \mathbf{u}}{\partial x^{\alpha}}=\mathbf{Q}_{\alpha}-\mathbf{P}_{\alpha} .
$$

In order to describe the stress state at a point $\mathbf{Q}$, a parallelogram with the edges $d x^{1} \mathbf{Q}_{1}$ and $d x^{2} \mathbf{Q}_{2}$ is shown in Figure 1 . Define

$$
\mathbf{T}^{\alpha}=\frac{\mathrm{d} \mathbf{T}^{\alpha}}{\mathrm{d} x^{\alpha+1}}, \quad \mathrm{~d} x^{\alpha} \longrightarrow 0,
$$

where $3=1$ for indexes. Quantities $\mathbf{T}^{\alpha}(\alpha=1,2)$ are called the base forces at point $\mathbf{Q}$ in the two-dimensional coordinate system $x^{\alpha}$.

The $\mathbf{T}^{\alpha}$ and $\mathbf{u}_{\alpha}$ can directly be expressed by strain energy. Thus, $\mathbf{u}_{\alpha}$ is just the conjugate variable of $\mathbf{T}^{\alpha}$. It can be seen that the mechanics problem can be completely established by means of $\mathbf{T}^{\alpha}$ and $\mathbf{u}_{\alpha}$.

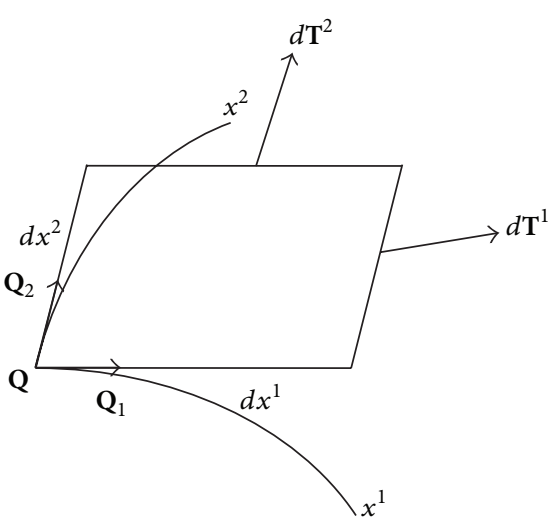

Figure 1: Base forces on plane problem.

According to the definitions of Cauchy stress tensors, the relation between the base forces and Cauchy stress tensors can be given as

$$
\boldsymbol{\sigma}=A_{\mathrm{Q}}^{-1} \mathbf{T}^{\alpha} \otimes \mathbf{Q}_{\alpha},
$$

where $\otimes$ is the dyadic symbol, the summation rule is implied, and $A_{Q}$ is the current base area

$$
A_{Q}=\left|\mathbf{Q}_{1} \times \mathbf{Q}_{2}\right| .
$$

For the isotropic material, the complementary energy density can be expressed as follows:

$$
W_{\mathrm{C}}=(2 E)^{-1}\left[(1+v) J_{2 T}-v J_{1 T}^{2}\right]
$$

in which $E$ is Young's modulus, $v$ is Poisson's ratio, and $J_{1 T}$ and $J_{2 T}$ are the invariants of $\mathbf{T}^{\alpha}$, so

$$
J_{1 T}=A_{P}^{-1} \mathbf{T}^{\alpha} \cdot \mathbf{P}_{\alpha}, \quad J_{2 T}=\left(A_{P}^{2}\right)^{-1}\left(\mathbf{T}^{\alpha} \cdot \mathbf{T}^{\beta}\right) p_{\alpha \beta},
$$

where $A_{P}$ is the original base area, $p_{\alpha \beta}$ is a metric tensor in the original configurations, and there are the following relations:

$$
\begin{gathered}
A_{P}=\left|\mathbf{P}_{1} \times \mathbf{P}_{2}\right|, \\
p_{\alpha \beta}=\mathbf{P}_{\alpha} \cdot \mathbf{P}_{\beta} .
\end{gathered}
$$

\section{Compliance Matrix}

Now, consider a 4-mid-node plane element as shown in Figure 2. Let $I, J, K$, and $L$ denote its edges and $\mathbf{T}^{I}, \mathbf{T}^{J}$, $\mathbf{T}^{K}$, and $\mathbf{T}^{L}$ the force vectors acting on each of the edges.

Substituting (8) into (7), the complementary energy of an element can be reduced as follows:

$$
W_{C}^{e}=\frac{1+v}{2 E A}\left[\left(\mathbf{T}^{I} \cdot \mathbf{T}^{J}\right) p_{I J}-\frac{v}{1+v}\left(\mathbf{T}^{I} \cdot \mathbf{P}_{\mathbf{I}}\right)^{2}\right],
$$

where $A$ is the original area of the element, $\mathbf{T}^{I}$ is the force vectors acting on the center of the boundary edge $I$ of the element, $p_{I J}$ is the dot product of position vectors $\mathbf{P}_{I}$ and $\mathbf{P}_{J}$ of points $I$ and $J$, and there are

$$
\begin{gathered}
\mathbf{T}^{I}=T^{I 1} \mathbf{e}_{1}+T^{I 2} \mathbf{e}_{2}, \quad \mathbf{T}^{J}=T^{J 1} \mathbf{e}_{1}+T^{J 2} \mathbf{e}_{2} \\
p_{I J}=\mathbf{P}_{I} \cdot \mathbf{P}_{\mathbf{J}}, \quad \mathbf{P}_{I}=P_{I 1} \mathbf{e}_{1}+P_{I 2} \mathbf{e}_{2}, \\
\mathbf{P}_{J}=P_{J 1} \mathbf{e}_{1}+P_{J 2} \mathbf{e}_{2}
\end{gathered}
$$

in which $\mathbf{e}_{1}$ and $\mathbf{e}_{2}$ are the unit vectors. 


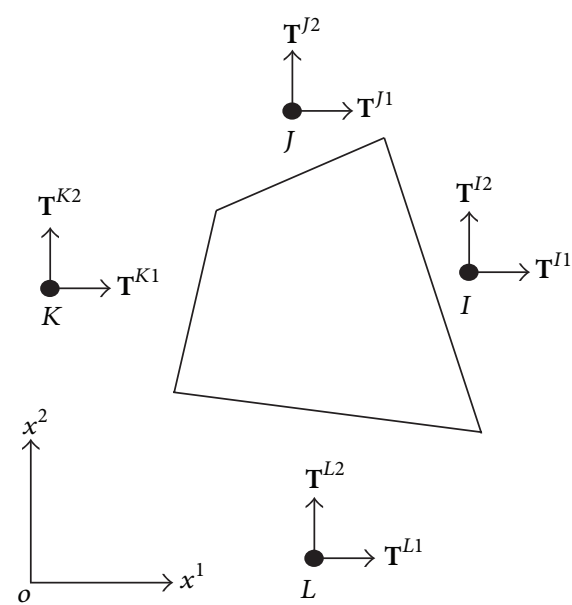

Figure 2: Four-mid-node plane element.

From (10), we can obtain the generalized displacement corresponding to $\mathrm{T}^{I}$

$$
\delta_{I}=\frac{\partial W_{C}^{e}}{\partial \mathbf{T}^{I}}=\mathbf{C}_{I J} \cdot \mathbf{T}^{J}
$$

where $\mathbf{C}_{I J}$ is a second-order tensor that is called the compliance matrix

$$
\mathbf{C}_{I J}=\frac{1+v}{E A}\left(p_{I J} \mathbf{U}-\frac{v}{1+v} \mathbf{P}_{I} \otimes \mathbf{P}_{J}\right) \quad(I, J=1,2,3,4),
$$

where $\mathbf{U}$ is the unit tensor, and it can be expressed as

$$
\mathbf{U}=\mathbf{e}_{1} \otimes \mathbf{e}_{1}+\mathbf{e}_{2} \otimes \mathbf{e}_{2} .
$$

Substituting (12) and (15) into (14), the compliance matrix of an element can be reduced as follows:

$$
\begin{aligned}
\mathbf{C}_{I J}=\frac{1+v}{E A}[ & \left(\frac{1}{1+v} P_{I 1} P_{J 1}+P_{I 2} P_{J 2}\right) \mathbf{e}_{1} \otimes \mathbf{e}_{1} \\
& -\frac{v}{1+v} P_{I 1} P_{J 2} \mathbf{e}_{1} \otimes \mathbf{e}_{2}-\frac{v}{1+v} P_{I 2} P_{J 1} \mathbf{e}_{2} \otimes \mathbf{e}_{1} \\
& \left.+\left(P_{I 1} P_{J 1}+\frac{1}{1+v} P_{I 2} P_{J 2}\right) \mathbf{e}_{2} \otimes \mathbf{e}_{2}\right]
\end{aligned}
$$

or

$$
\begin{aligned}
\mathbf{C}_{I J}= & C_{I 1 J 1} \mathbf{e}_{1} \otimes \mathbf{e}_{1}+C_{I 1 J 2} \mathbf{e}_{1} \otimes \mathbf{e}_{2} \\
& +C_{I 2 J 1} \mathbf{e}_{2} \otimes \mathbf{e}_{1}+C_{I 2 J 2} \mathbf{e}_{2} \otimes \mathbf{e}_{2},
\end{aligned}
$$

or

$$
\begin{aligned}
\mathrm{C}_{I J}= & \frac{1+v}{E A} \\
\times & {\left[\begin{array}{cc}
\frac{1}{1+v} P_{I 1} P_{J 1}+P_{I 2} P_{J 2} & -\frac{v}{1+v} P_{I 1} P_{J 2} \\
-\frac{v}{1+v} P_{I 2} P_{J 1} & P_{I 1} P_{J 1}+\frac{1}{1+v} P_{I 2} P_{J 2}
\end{array}\right] } \\
& (I, J=1,2,3,4) .
\end{aligned}
$$

For a strain problem, it is necessary to replace $E$ with $E /\left(1-v^{2}\right)$ and $\nu$ with $v /(1-v)$ in (16)-(18).

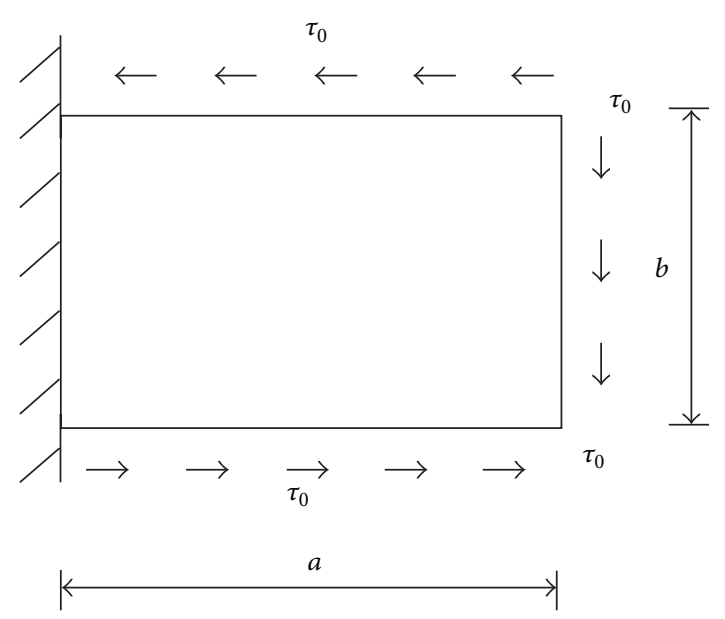

FIGURE 3: Rectangular plate subjected to shearing forces.

\section{Governing Equations}

The total complementary energy of the elastic system can be written as

$$
\Pi_{C}=\sum_{n}\left(W_{C}^{e}-\overline{\mathbf{u}}_{I} \cdot \mathbf{T}^{I}\right)
$$

According to the complementary energy principle, $\Pi_{C}^{\text {ne }}$ takes stationary value under equilibrium conditions and stress boundary conditions for the real displacement and stress state.

The equilibrium conditions can be released by the Lagrange multiplier method. For the stress boundary condition and the corresponding condition of the face forces between elements, computational techniques can be applied to achieve a rotation. Using the Lagrange multiplier method, a new function for an element can be introduced as follows:

$$
\begin{aligned}
\Pi_{C}^{e^{*}}\left(\mathbf{T}, \lambda, \lambda_{3}\right)= & \Pi_{C}^{e}(\mathbf{T}) \\
& +\lambda\left(\sum_{I=1}^{4} \mathbf{T}^{I}\right)+\lambda_{3}\left(\mathbf{T}^{I} \times \mathbf{P}_{I}\right)
\end{aligned}
$$

in which arbitrary vectors $\lambda, \lambda_{3}$ are the Lagrange multipliers, and $\lambda$ can be expressed as

$$
\lambda=\lambda_{1} \mathbf{e}_{1}+\lambda_{2} \mathbf{e}_{2}
$$

For the elastic system, there is

$$
\Pi_{C}^{*}=\sum_{n}\left[\Pi_{C}^{e^{*}}\left(\mathbf{T}, \lambda, \lambda_{3}\right)\right]
$$

And by means of the modified complementary energy principle

$$
\delta \Pi_{C}^{*}=\sum_{n}\left[\delta \Pi_{C}^{e^{*}}\left(\mathbf{T}, \lambda, \lambda_{3}\right)\right]=0 .
$$




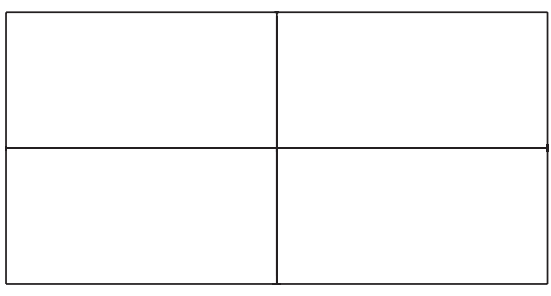

(a) Uniform 4-side elements

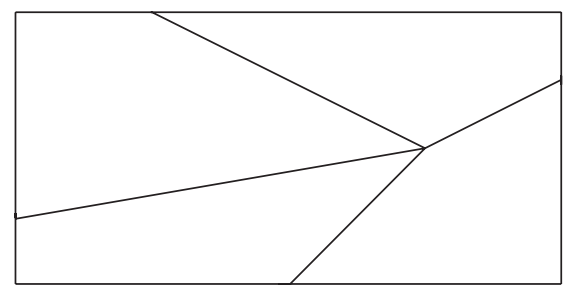

(b) Arbitrary 4-side elements

Figure 4: Two kinds of meshes for the pure shear problem.

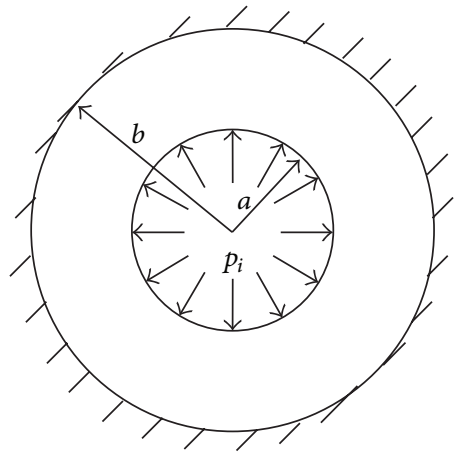

FIGURE 5: Thick cylinder with fixed outer edge under internal pressure.

Further, (23) can be expressed as

$$
\begin{gathered}
\frac{\partial \Pi_{C}^{*}\left(\mathbf{T}, \lambda, \lambda_{3}\right)}{\partial \mathbf{T}}=0, \quad \frac{\partial \Pi_{C}^{*}\left(\mathbf{T}, \lambda, \lambda_{3}\right)}{\partial \lambda}=0, \\
\frac{\partial \Pi_{C}^{*}\left(\mathbf{T}, \lambda, \lambda_{3}\right)}{\partial \lambda_{3}}=0 .
\end{gathered}
$$

Equations (24) are the compatibility equations and displacement boundary conditions for the elastic system. These are the governing equations of the BFEM.

An explicit expression of stress for the 4-mid-node element, according to (5), can be written as

$$
\sigma=A^{-1} \mathbf{T}^{I} \otimes \mathbf{P}_{I}
$$

or

$$
\begin{aligned}
\sigma=A^{-1} \sum_{I=1}^{4}[ & T^{I 1} P_{I 1} \mathbf{e}_{1} \otimes \mathbf{e}_{1}+T^{I 1} P_{I 2} \mathbf{e}_{1} \otimes \mathbf{e}_{2} \\
& \left.+T^{I 2} P_{I 1} \mathbf{e}_{2} \otimes \mathbf{e}_{1}+T^{I 2} P_{I 2} \mathbf{e}_{2} \otimes \mathbf{e}_{2}\right]
\end{aligned}
$$

or

$$
\sigma=\frac{1}{A}\left[\begin{array}{ll}
\sum_{I=1}^{4} T^{I 1} P_{I 1} & \sum_{I=1}^{4} T^{I 1} P_{I 2} \\
\sum_{I=1}^{4} T^{I 2} P_{I 1} & \sum_{I=1}^{4} T^{I 2} P_{I 2}
\end{array}\right] .
$$

An explicit expression for displacement of node, based on the governing equation of element, can be developed.
For a 4-mid-node plane element, the governing equation can be written as

$$
\delta_{I}=\frac{\partial \Pi_{C}^{e *}\left(\mathrm{~T}, \lambda, \lambda_{3}\right)}{\partial \mathbf{T}^{I}} .
$$

Substituting (20) into (28) and considering (19), (10), and (14), the explicit expression for displacement of nodes can be written as

$$
\delta_{I}=\mathbf{C}_{I J} \cdot \mathbf{T}^{J}+\lambda+\lambda_{3} \varepsilon \cdot \mathbf{P}_{I}
$$

where $\varepsilon$ is the alternating tensor and $\varepsilon$ can be written as

$$
\varepsilon=\varepsilon^{\alpha \beta} \mathbf{e}_{\alpha} \otimes \mathbf{e}_{\beta}
$$

or

$$
\varepsilon=\mathbf{e}_{1} \otimes \mathbf{e}_{2}-\mathbf{e}_{2} \otimes \mathbf{e}_{1} .
$$

Substituting (11), (17), (21), and (31) into (29), the explicit expression for displacement of nodes can be written as

$$
\begin{aligned}
\delta_{I}= & \left(C_{I 1 J 1} T^{J 1}+C_{I 1 J 2} T^{J 2}+\lambda_{1}+\lambda_{3} P_{I 2}\right) \mathbf{e}_{1} \\
& +\left(C_{I 2 J 1} T^{J 1}+C_{I 2 J 2} T^{J 2}+\lambda_{2}-\lambda_{3} P_{I 1}\right) \mathbf{e}_{2}
\end{aligned}
$$

or

$$
\delta_{I}=\left\{\begin{array}{l}
C_{I 1 J 1} T^{J 1}+C_{I 1 J 2} T^{J 2}+\lambda_{1}+\lambda_{3} P_{I 2} \\
C_{I 2 J 1} T^{J 1}+C_{I 2 J 2} T^{J 2}+\lambda_{2}-\lambda_{3} P_{I 1}
\end{array}\right\} .
$$

\section{Numerical Examples}

To verify the validity and accuracy of the 4-mid-node plane model of base force element method proposed in this paper, a program on the BFEM is made, and a number of the numerical examples on the BFEM for the typical plane elasticity problems are presented in this section. The results obtained from the 2D BFEM are compared with corresponding theoretical solutions. The responses obtained using the traditional displacement finite element method are also given for some problems in order to assess the advantages of the 2D BFEM. All calculations of the traditional displacement finite element method are performed using the four-node isoparametric element (Q4 model).

Example 1 (pure shear of rectangular plate). Consider the problem of a rectangular plate subjected to uniformly distributing shearing forces as shown in Figure 3. Parameters about 


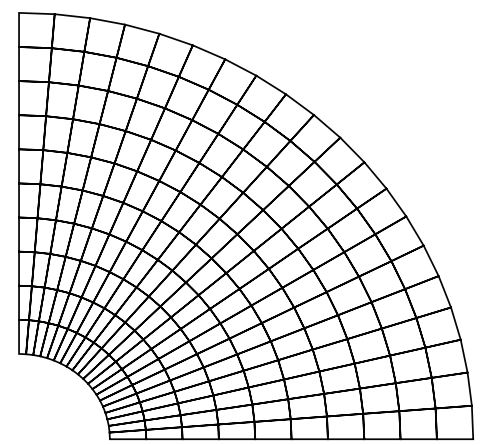

(a) $20 \times 10$ elements

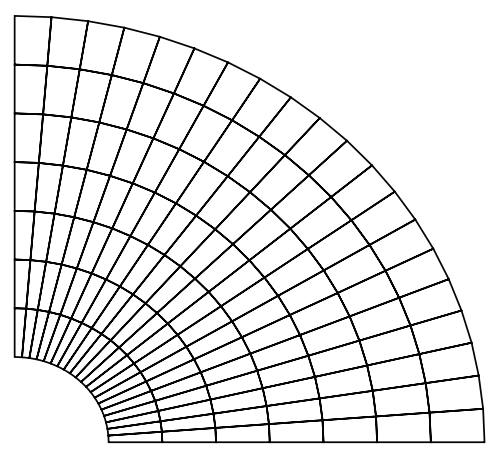

(b) $20 \times 7$ elements

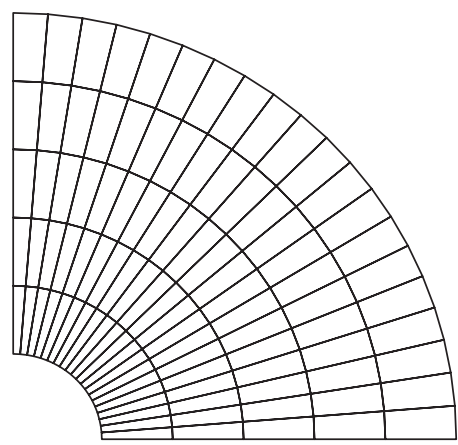

(c) $20 \times 5$ elements

FIgURE 6: Three kinds of meshes for cylinder.

TABLE 1: The values of $\sigma_{r}$.

\begin{tabular}{lcccc}
\hline \multirow{2}{*}{ Meshes } & Location & \multicolumn{3}{c}{$\sigma_{r} /(\mathrm{MPa})$} \\
& & $\mathrm{Q} 4$ & BFEM & Theoretical \\
\hline $20 \times 10$ & $r=2.8000$ & -0.2066 & -0.2072 & -0.2069 \\
$20 \times 7$ & $r=3.0000$ & -0.1914 & -0.1926 & -0.1919 \\
$20 \times 5$ & $r=3.0000$ & -0.1912 & -0.1935 & -0.1919 \\
\hline
\end{tabular}

TABLE 2: The values of $\sigma_{\theta}$.

\begin{tabular}{lcccc}
\hline \multirow{2}{*}{ Meshes } & Location & \multicolumn{3}{c}{$\sigma_{\theta} /(\mathrm{MPa})$} \\
& & Q4 & BFEM & Theoretical \\
\hline $20 \times 10$ & $r=2.8000$ & 0.0274 & 0.0253 & 0.0250 \\
$20 \times 7$ & $r=3.0000$ & 0.0113 & 0.0107 & 0.0101 \\
$20 \times 5$ & $r=3.0000$ & 0.0124 & 0.0116 & 0.0101 \\
\hline
\end{tabular}

the calculated specimen are taken as $a=2.0 \mathrm{~m}, b=1.0 \mathrm{~m}$, $E=10^{5} \mathrm{MPa}, \nu=0.3$, and $\tau_{0}=1.0 \mathrm{MPa}$ for a plane stress problem. The calculation is done using two different element meshes with the center nodes of each of the edges as shown in Figure 4 successively.

The numerical solutions are that the stress component of various elements is $\tau_{x y}=1$ for two kinds of different meshes. The results are consistent with the theoretical results [1].

Example 2 (thick cylinder with fixed outer surface under internal pressure). Consider a thick cylinder with inner radius $a$ and outer radius $b$ under inner pressure $p_{i}$ as shown in Figure 5.

The calculated parameters are taken to be $a=1.0 \mathrm{~m}, b=$ $5.0 \mathrm{~m}, E=10^{6} \mathrm{MPa}, v=0.3$, and $p_{i}=1.0 \mathrm{MPa}$ for a plane strain problem. The calculation is done using three different element meshes with the center nodes of each of the edges as shown in Figure 6 successively.

The values of stress components $\sigma_{r}, \sigma_{\theta}$, and $u_{r}$ for various radiuses $r$ are listed in Tables 1, 2, and 3, respectively. Comparisons of the results from the theoretical solution [1] and traditional 4-node quadrilateral isoparametric element (Q4 model) are also given in Tables 1, 2, and 3, respectively. The numerical results of the present model are consistent with those of the theoretical solutions and have given very good performance compared with Q4 model.

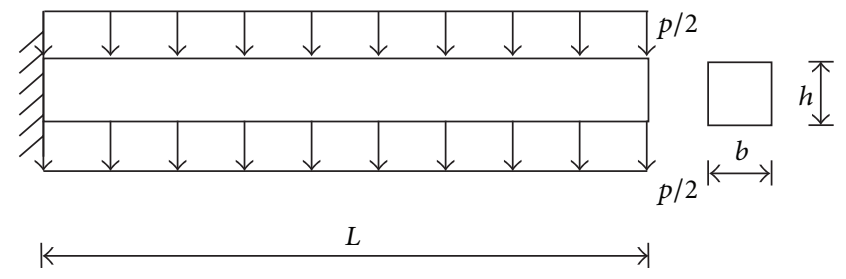

FIGURE 7: Cantilever beam under uniformly distributed load.

Example 3 (a cantilever under uniformly distributed load). It consists of a straight cantilever beam under uniformly distributed load, as shown in Figure 7. The beam has length $L=$ $10 \mathrm{~m}$, cross-sectional dimensions $b=1 \mathrm{~m}$ and $h=1 \mathrm{~m}$, elastic modulus $E=10^{9} \mathrm{MPa}$, and Poisson's ratio $v=0$, respectively. While the applied total load level is $p=10 \mathrm{MPa}$. The calculated specimen was divided into the 4-mid-node base force elements with the center nodes of each of the edges, as shown in Figure 8.

The vertical displacement solutions of BFEM at the tip of the beam with different meshes are listed in Table 4 . The vertical displacement of BFEM at the tip of the beam is compared with that provided by traditional 4-node quadrilateral isoparametric element (Q4 model) and the analytical solution.

Table 4 shows that the results of the present formulation agree well with those of the analytical solution, and the 4mid-node element of BFEM has given very good performance compared with Q4 model for the large aspect ratio of elements.

Example 4 (a cantilever with large aspect ratio under a concentrated end load). A clamped cantilever with a concentrated end load is carried out as shown in Figure 9. The beam has length $L=5 \mathrm{~m}$, cross-sectional dimensions $b=1 \mathrm{~m}$ and $h=0.1 \mathrm{~m}$, elastic modulus $E=10^{9} \mathrm{~N} / \mathrm{m}^{2}$, and Poisson's ratio $v=0$. While the applied total load level is $P=1 \mathrm{~N}$. The calculated specimen was divided into the 4-mid-node base force elements with the center nodes of each of the edges, as shown in Figure 10.

The vertical displacement of BFEM at the tip of the beam is compared with that provided by traditional 4-node quadrilateral isoparametric element (Q4 model) and the analytical 


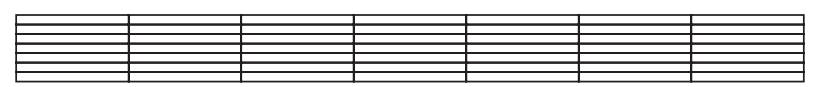

(a) $7 \times 7$ elements

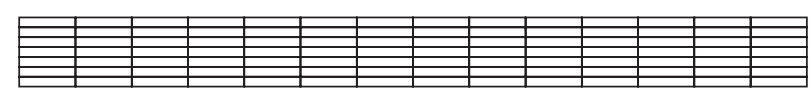

(b) $14 \times 7$ elements

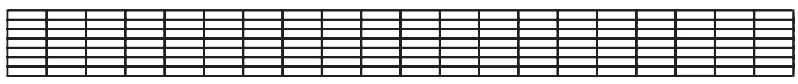

(c) $20 \times 7$ elements

FIgURE 8: Meshes of cantilever beam under uniformly distributed load.

TABLE 3: The values of $u_{r}$.

\begin{tabular}{lcccc}
\hline \multirow{2}{*}{ Meshes } & Location & \multicolumn{3}{c}{$u_{r} /\left(\times 10^{-6} \mathrm{~m}\right)$} \\
& & $\mathrm{Q} 4$ & BFEM & Theoretical \\
\hline $20 \times 10$ & $r=1.0000$ & 1.1274 & 1.1340 & 1.1346 \\
$20 \times 7$ & $r=1.0000$ & 1.1212 & 1.1340 & 1.1346 \\
$20 \times 5$ & $r=1.0000$ & 1.1113 & 1.1340 & 1.1346 \\
\hline
\end{tabular}

TABLE 4: Vertical displacement of the cantilever beam under uniformly distributed load.

\begin{tabular}{lccc}
\hline Meshes & & $v\left(\times 10^{-4}\right)$ & \\
& BFEM & Q4 model & Analytical \\
\hline $20 \times 7$ & 1.4441 & 1.3447 & 1.5000 \\
$14 \times 7$ & 1.4041 & 1.2062 & 1.5000 \\
$7 \times 7$ & 1.2802 & 0.7528 & 1.5000 \\
\hline
\end{tabular}

TABLE 5: Vertical displacement of the cantilever beam with a concentrated end load.

\begin{tabular}{lccc}
\hline Meshes & & $v\left(\times 10^{-4}\right)$ & \\
& BFEM & Q4 model & Analytical \\
\hline $60 \times 9$ & -5.0633 & -3.7122 & -5.0000 \\
$30 \times 9$ & -5.0623 & -2.0935 & -5.0000 \\
$15 \times 9$ & -5.0581 & -0.7629 & -5.0000 \\
\hline
\end{tabular}

TABLE 6: Normalized vertical displacement with the effect of mesh sensitivity.

\begin{tabular}{lcccc}
\hline Mesh distortion parameters $d$ & 0.0 & 0.5 & 1.0 & 1.5 \\
\hline BFEM & 1.0058 & 1.0014 & 0.9868 & 0.9654 \\
Q4 & 0.0744 & 0.0463 & 0.0380 & 0.0361 \\
\hline
\end{tabular}

solution in Table 5. The results show that the 4-mid-node element of BFEM has given very good performance compared with Q4 model for the large aspect ratio of elements.

Example 5 (a cantilever with mesh distortion under a concentrated end load). A clamped cantilever with a concentrated end load is carried out as shown in Figure 9. The beam has length $L=10 \mathrm{~m}$, cross-sectional dimensions $b=1 \mathrm{~m}$ and $h=1 \mathrm{~m}$, elastic modulus $E=10^{6} \mathrm{~Pa}$, and Poisson's ratio $v=0$, while the applied total load level is $P=1 \mathrm{~N}$. The calculated specimen was divided into the 4-mid-node base force elements with the center nodes of each of the edges, as shown in Figure 11.

The normalized vertical displacement of BFEM at the tip of the beam is compared with that provided by traditional

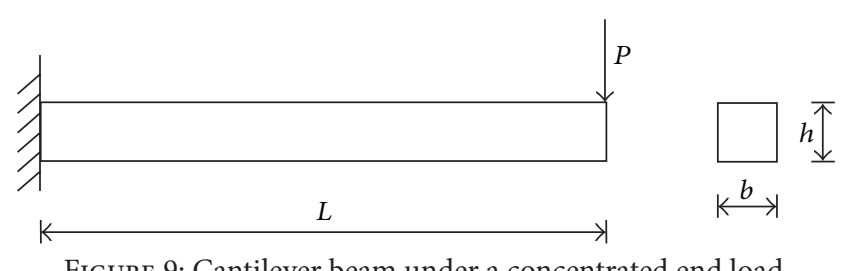

Figure 9: Cantilever beam under a concentrated end load.

4-node quadrilateral isoparametric element (Q4 model) and the analytical solution in Table 6 . The results show that the 4-mid-node element of BFEM has given very good performance compared with Q4 model for the distortion mesh problems.

\section{Conclusions}

In this paper, a 4-mid-node plane model of base force element method (BFEM) on complementary energy principle is proposed for elasticity problems. Based on the complementary energy principle, the equilibrium conditions are released by the Lagrange multiplier method, and a modified complementary energy principle described by the base forces is obtained. The 4-mid-node plane element model is derived by assuming that the stress is uniformly distributed on each edge of a plane element. A BFEM procedure is developed using MATLAB language. The chief features of the method are that the model does not introduce an interpolating function and can be used in any coordinate system and is not necessary to introduce Gauss' integral for calculating the stiffness coefficient at a point.

The present model is applied for the solution of some elasticity problems. The comparisons are made with the corresponding analytical solutions, and a good agreement of results is observed. A series of numerical experiments are performed in order to assess the relative performances of the present model; based on these studies, it is found that the 4mid-node plane model of BFEM with complementary energy principle provides reliable predictions for elasticity problems. A good performance of the 4-mid-node plane element of BFEM is observed for some problems.

The 4-mid-node plane element model of BFEM is also compared with the standard displacement method. It has been demonstrated by the results of numerical calculation that the new BFEM formulations proposed in this paper are effective and accurate. The 4-mid-node plane element model can be used efficiently for the elasticity analysis with increasing element aspect ratios and distortion meshes. The results presented here revealed a better overall performance of 


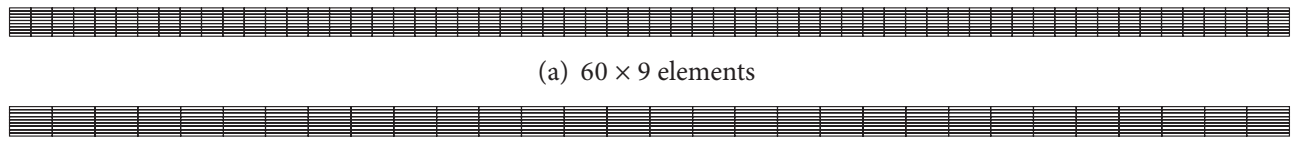

(b) $30 \times 9$ elements

(c) $15 \times 9$ elements

FIGURE 10: Meshes of cantilever beam under a concentrated end load.

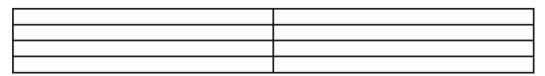

(a) Mesh distortion parameters $d=0$

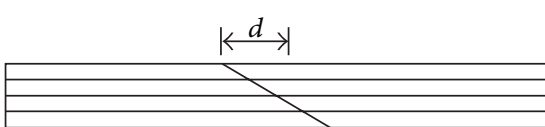

(b) Mesh distortion parameters $d=0.5$

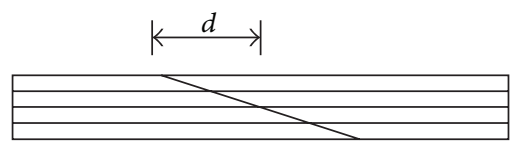

(d) Mesh distortion parameters $d=1.5$

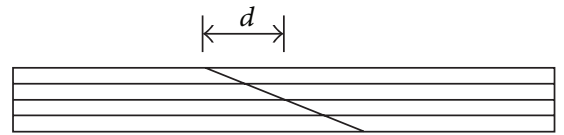

(c) Mesh distortion parameters $d=1.0$

FIGURE 11: Distortion meshes of cantilever beam.

the BFEM in calculations. These results provide further confirmation that the BFEM can be used successfully and efficiently in the structural analysis, thus serving as an alternative method, and certain applications, being the method of choice for the analysis.

\section{Conflict of Interests}

The authors declare that there is no conflict of interests.

\section{Acknowledgment}

This work is supported by the National Science Foundation of China (nos. 91016026, 10972015, and 11172015).

\section{References}

[1] O. C. Zienkiewicz, The Finite Element Method, McGraw-Hill, New York, NY, USA, 1977.

[2] K. J. Bathe, Finite Element Procedures in Engineering Analysis, Prentice Hall, Upper Saddle River, NJ, USA, 1982.

[3] K. J. Bathe, Finite Element Procedures, Prentice Hall, Upper Saddle River, NJ, USA, 1996.

[4] R. D. Cook, Concepts and Applications of Finite Element Analysis, Wiley, New York, NY, USA, 1981.

[5] H. A. F. A. Santos and J. P. Moitinho de Almeida, "Equilibriumbased finite-element formulation for the geometrically exact analysis of planar framed structures," Journal of Engineering Mechanics, vol. 136, no. 12, pp. 1474-1490, 2010.

[6] H. A. F. A. Santos, "Complementary-energy methods for geometrically non-linear structural models: an overview and recent developments in the analysis of frames," Archives of Computational Methods in Engineering, vol. 18, no. 4, pp. 405-440, 2011.

[7] H. A. F. A. Santos and C. I. Almeida Paulo, "On a pure complementary energy principle and a force-based finite element formulation for non-linear elastic cables," International Journal of Non-Linear Mechanics, vol. 46, no. 2, pp. 395-406, 2011.
[8] X.-R. Fu, S. Cen, C. F. Li, and X.-M. Chen, "Analytical trial function method for development of new 8-node plane element based on the variational principle containing airy stress function," Engineering Computations, vol. 27, no. 4, pp. 442-463, 2010.

[9] S. Cen, X. Fu, G. Zhou, M. Zhou, and C. Li, "Shape-free finite element method: the plane hybrid stress-function (HS-F) element method for anisotropic materials," Science China, vol. 54, no. 4, pp. 653-665, 2011.

[10] S. Cen, X.-R. Fu, and M.-J. Zhou, "8- and 12-node plane hybrid stress-function elements immune to severely distorted mesh containing elements with concave shapes," Computer Methods in Applied Mechanics and Engineering, vol. 200, no. 29-32, pp. 2321-2336, 2011.

[11] S. Cen, M.-J. Zhou, and X.-R. Fu, "A 4-node hybrid stress-function (HS-F) plane element with drilling degrees of freedom less sensitive to severe mesh distortions," Computers and Structures, vol. 89, no. 5-6, pp. 517-528, 2011.

[12] S. Cen, G.-H. Zhou, and X.-R. Fu, "A shape-free 8-node plane element unsymmetric analytical trial function method," International Journal for Numerical Methods in Engineering, vol. 91, pp. 158-185, 2012.

[13] Y. C. Gao, "A new description of the stress state at a point with applications," Archive of Applied Mechanics, vol. 73, no. 3-4, pp. 171-183, 2003.

[14] Y. C. Gao and T. J. Gao, "Large deformation contact of a rubber notch with a rigid wedge," International Journal of Solids and Structures, vol. 37, no. 32, pp. 4319-4334, 2000.

[15] Y. C. Gao, "Analysis of the interface crack for rubber-like materials," Journal of Elasticity, vol. 66, no. 1, pp. 1-19, 2002.

[16] Y. J. Peng, Z. L. Dong, B. Peng, and Y. H. Liu, "Base force element method (BFEM) on potential energy principle for elasticity problems," International Journal of Mechanics and Materials in Design, vol. 7, no. 3, pp. 245-251, 2011.

[17] Y. J. Peng and Y. H. Liu, "Base force element method of complementary energy principle for large rotation problems," Acta Mechanica Sinica, vol. 25, no. 4, pp. 507-515, 2009. 
[18] Y. H. Liu and Y. J. Peng, "Base force element method (BFEM) on complementary energy principle for linear elasticity problem," Science China, vol. 54, no. 11, pp. 2025-2032, 2011.

[19] Y. J. Peng, Z. L. Dong, B. Peng, and N. N. Zong, "The application of $2 \mathrm{D}$ base force element method (BFEM) to geometrically nonlinear analysis," International Journal of Non-Linear Mechanics, vol. 47, no. 3, pp. 153-161, 2012. 


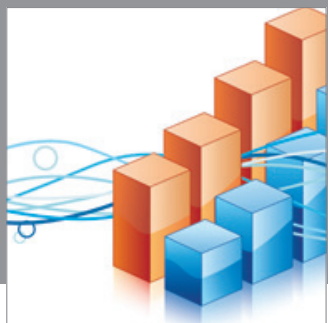

Advances in

Operations Research

mansans

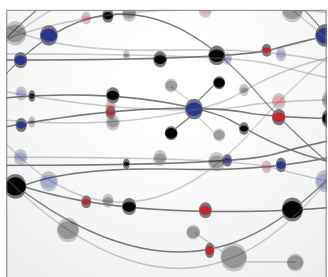

The Scientific World Journal
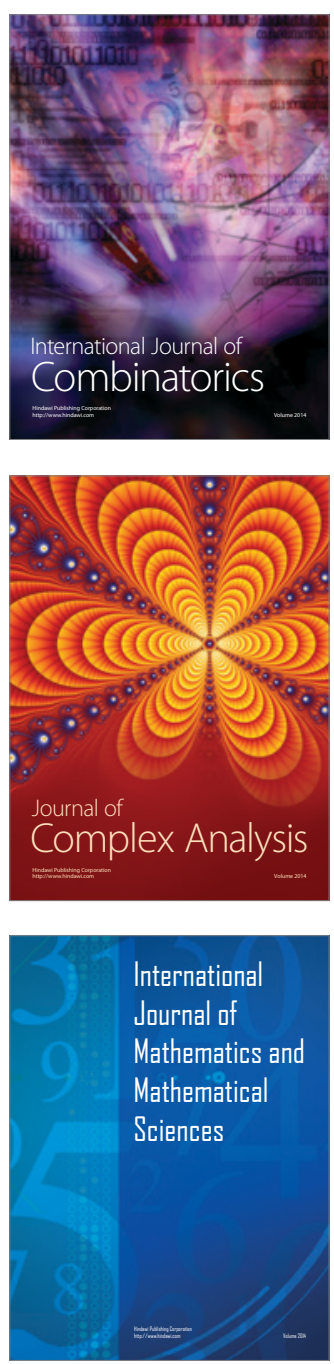
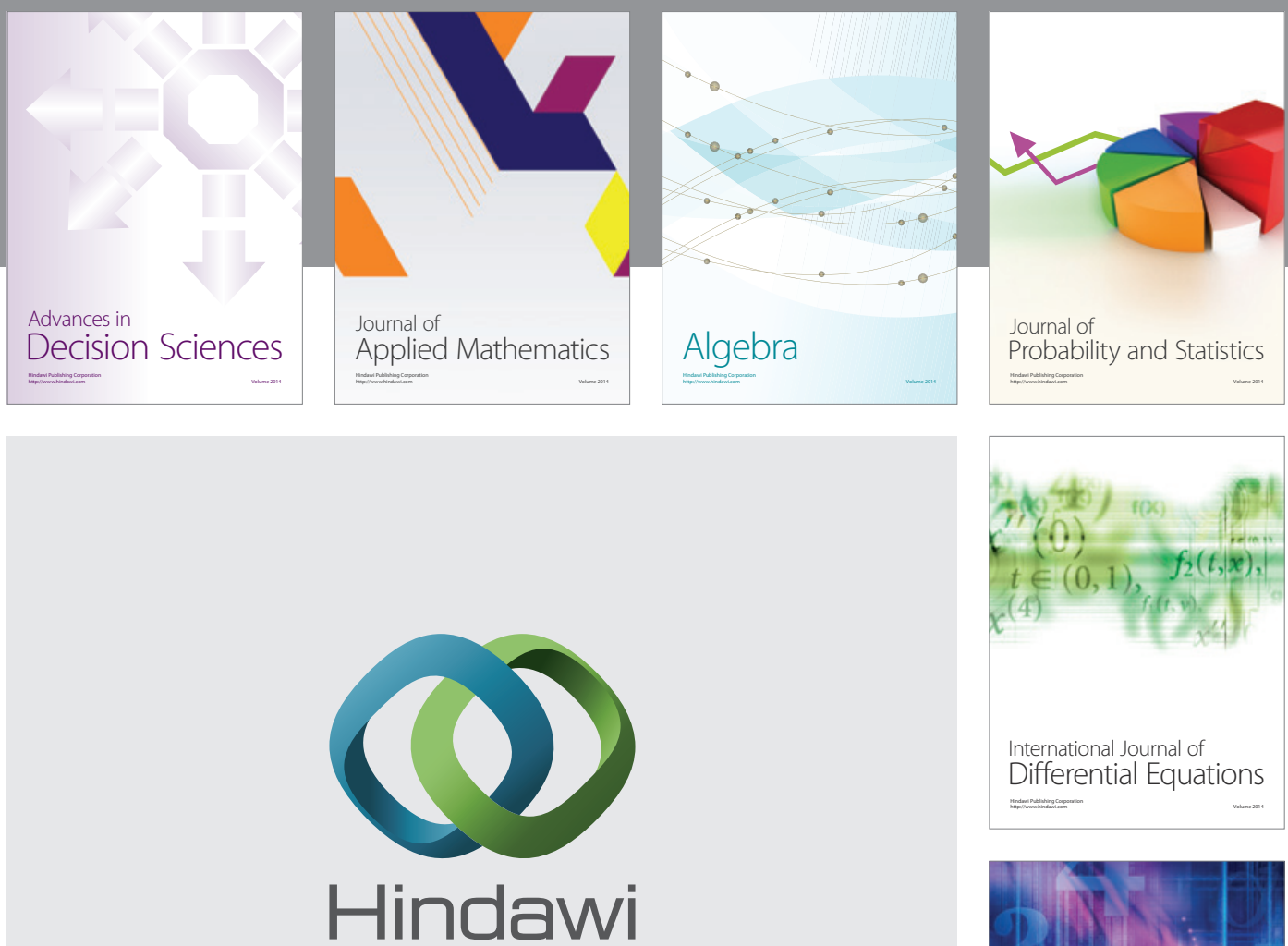

Submit your manuscripts at http://www.hindawi.com
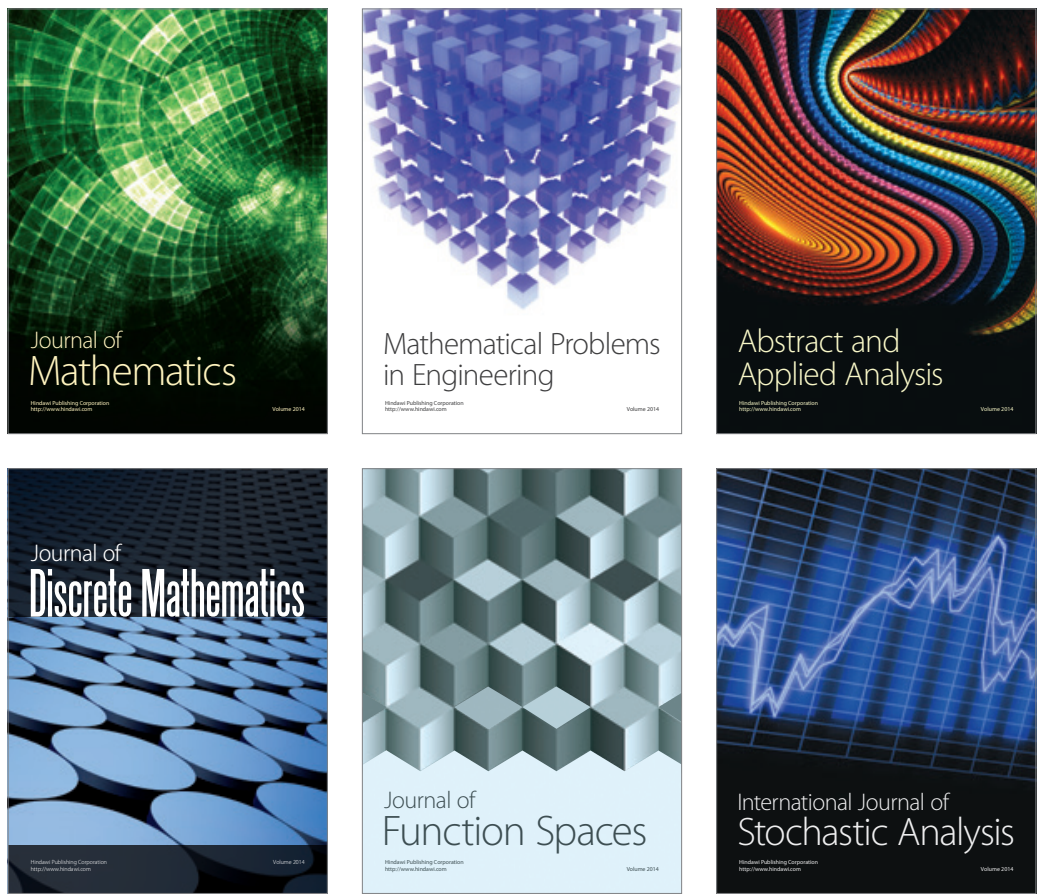

Journal of

Function Spaces

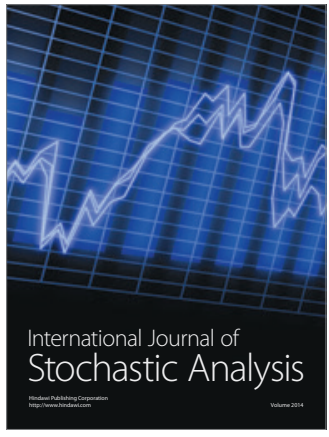

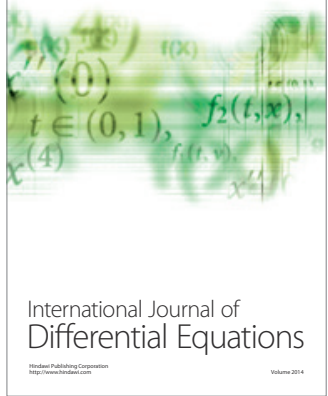
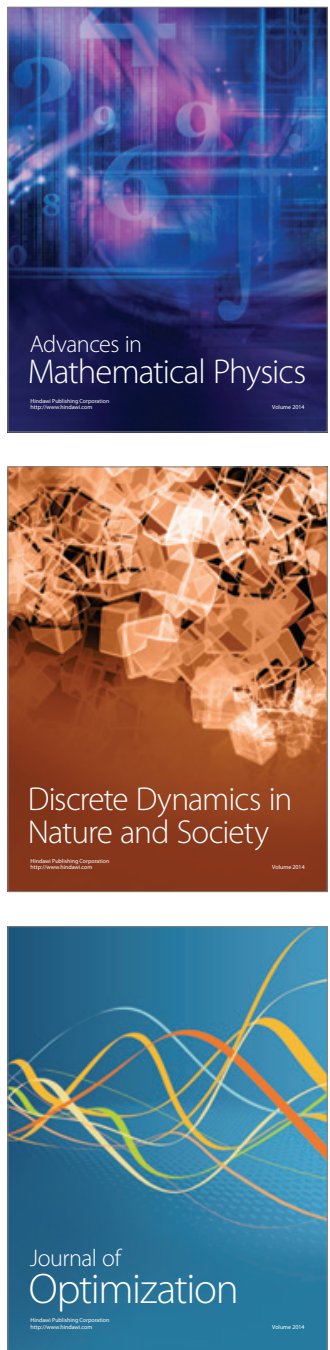